\title{
Spectral and kinetic features of thermoluminescence in hexagonal boron nitride powder after UV-irradiation
}

\author{
I.A. Weinstein*, A.S. Vokhmintsev, M.G. Minin, V.V. Kartashov, I.V. Chernetsky \\ Ural Federal University, Research and Educational Center "Nanomaterials and Nanotechnologies", Mira Street 19, Ekaterinburg 620002, Russia
}

\section{H I G H L I G H T S}

- UV-irradiated h-BN powder is characterized by three TL peaks in different spectral regions.

- TL peaks at 380 and $600 \mathrm{~K}$ are caused by capture levels of one-boron and three-boron centers, respectively.

- Visible range emissions of vacancy centers and oxygen-boron complexes dominate in thermoluminescence of h-BN.

\section{A R T I C L E I N F O}

\section{Article history:}

Received 15 October 2012

Received in revised form

11 December 2012

Accepted 26 December 2012

\section{Keywords:}

Boron nitride

Nitrogen vacancy

Oxygen-boron complexes

General order kinetics

\begin{abstract}
A B S T R A C T
Thermally stimulated luminescence (TL) from UV-irradiated h-BN powder synthesized using carbamide technique was studied. Three TL peaks at $T_{\max }=380,500$ and $600 \mathrm{~K}$ during linear heating with $2 \mathrm{~K} / \mathrm{s}$ rate in RT $-773 \mathrm{~K}$ temperature range were observed. It was found that the 2.90 and $3.25 \mathrm{eV}$ emission bands, which were related with recombination centers on the basis of $V_{\mathrm{N}}$ and $\mathrm{BO}^{-}$-complexes, dominate in $\mathrm{TL}$ spectra of h-BN. Experimental TL glow curves were analyzed in terms of general order kinetics and energy parameters of responsible capture levels were estimated. It was shown, considering the independent data on the luminescent properties of hexagonal boron nitride in different structural states, that TL peaks at $380 \mathrm{~K}$ and $600 \mathrm{~K}$ were due to traps based on the one-boron and the three-boron centers with thermal depth $E_{\mathrm{A}}=0.7$ and $1.0 \mathrm{eV}$, respectively. The possible origin of the trap with $E_{\mathrm{A}}=1.6 \mathrm{eV}$, responsible for the TL peak at $500 \mathrm{~K}$, is discussed.
\end{abstract}

(C) 2013 Elsevier Ltd. All rights reserved.

\section{Introduction}

Unique electrical and physical properties (wide energy gap, $215 \mathrm{~nm}$ lasing effect etc.) along with high thermal, chemical and radiation stability make hexagonal boron nitride a prospective material for multifunctional optoelectronic applications (Ci et al., 2010; Kubota et al., 2007; Watanabe et al., 2004; Solozhenko et al., 2001). It is known that the induced luminescence response in the irradiated materials based on c-BN and h-BN under thermal and optical stimulation is observed within the visible and UV range photon energies (Museur et al., 2008; Trinkler et al., 2004; Galanov et al., 1989; Katzir et al., 1975). These potential dosimetric signals are caused by processes with participating of different lattice defects of intrinsic and extrinsic genesis. At present it is established that many features in the behavior of hexagonal and cubic boron nitrides exposed to radiation are dictated by the complexes based on nitrogen vacancies with substitutional oxygen atoms and

\footnotetext{
* Corresponding author. Tel.: +7 34337593 74; fax: +7 3433759373.

E-mail address: i.a.weinstein@ustu.ru (I.A. Weinstein).
}

carbonic impurities (Kubota et al., 2007; Museur et al., 2007; Trinkler et al., 2004). In addition, defects of synthesis or radiation origin have similar spectral parameters and are to be taken into account while analyzing induced luminescence processes. So the purpose of the present study is to investigate kinetic features of spectrally resolved thermoluminescence (TL) in the UV-irradiated h-BN powder.

\section{Experimental}

Hexagonal boron nitride samples synthesized using carbamide technique (Company "Unichim \& EP») have been studied. The powder under study had a typical flake-like morphology and consisted of $98.9 \mathrm{wt} . \%$ of boron nitride, $0.17 \mathrm{wt}$ \% of boron anhydride and $0.22 \mathrm{wt} . \%$ of carbon. Average size of particles was about $10 \mu \mathrm{m}$. Before the studies h-BN powder was annealed up to $773 \mathrm{~K}$. Fig. 1 shows the picture of the powder under study, obtained with scanning electronic microscope (Sigma VP Carl Zeiss, Inc.) with inlens detector, $\mathrm{EHT}=10 \mathrm{kV}$ and $\mathrm{WD}=1.4 \mathrm{~mm}$.

TL studies were performed on the laboratory installation based on Perkin Elmer LS 55 luminescence spectrometer with remote 


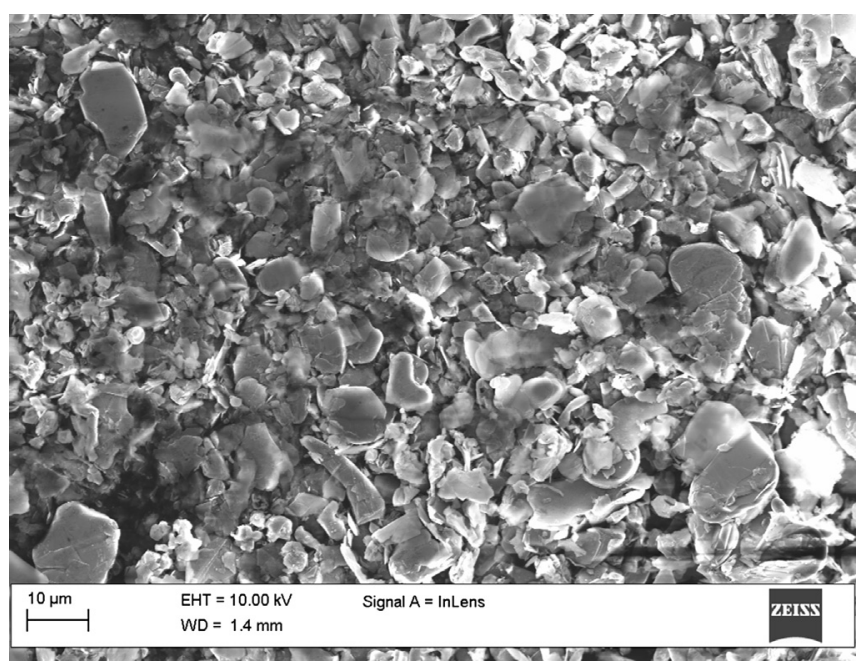

Fig. 1. The h-BN powder under study.

fiber optic accessory. h-BN powder was exposed to unfiltered UVradiation from the xenon discharge lamp for $5 \mathrm{~min}$. Thermally stimulated emission was measured during heating up to $773 \mathrm{~K}$ with constant rate $2 \mathrm{~K} / \mathrm{s}$. TL glow curves were recorded using an LS 55 emission monochromator and R8630 Hamamatsu photomultiplier tube within the $250-600 \mathrm{~nm}$ range and $25 \mathrm{~nm}$ increment.

To measure the TL spectra, the powder was exposed to unfiltered UV-radiation from the Xe lamp for 15 min. After that the powder was heated within the RT $-773 \mathrm{~K}$ range with the rate of $0.5 \mathrm{~K} / \mathrm{s}$. The TL spectra were recorded in every $30 \mathrm{~K}$ using an LS 55 emission monochromator and R8630 PMT within the 325-525 nm range and scanning rate of $800 \mathrm{~nm} / \mathrm{min}$. Temperature variation of the sample and luminescence intensity of a spectrum was neglected during the measuring time $(\approx 15 \mathrm{~s})$.

\section{Results and discussion}

Fig. 2 shows TL curves measured at different wavelengths. It is seen that the number of the TL peaks changes depending on spectral range. For example, near $425 \mathrm{~nm}$ three peaks are recorded with $T_{\max }=380 \mathrm{~K}, 500 \mathrm{~K}$ and $600 \mathrm{~K}(\mathrm{P} 1, \mathrm{P} 2$, and P3, respectively). The TL glow at $500 \mathrm{~K}$ dominates in this case. Two peaks are observed for $\lambda>475 \mathrm{~nm}$, while for $375 \mathrm{~nm}$ a high-temperature

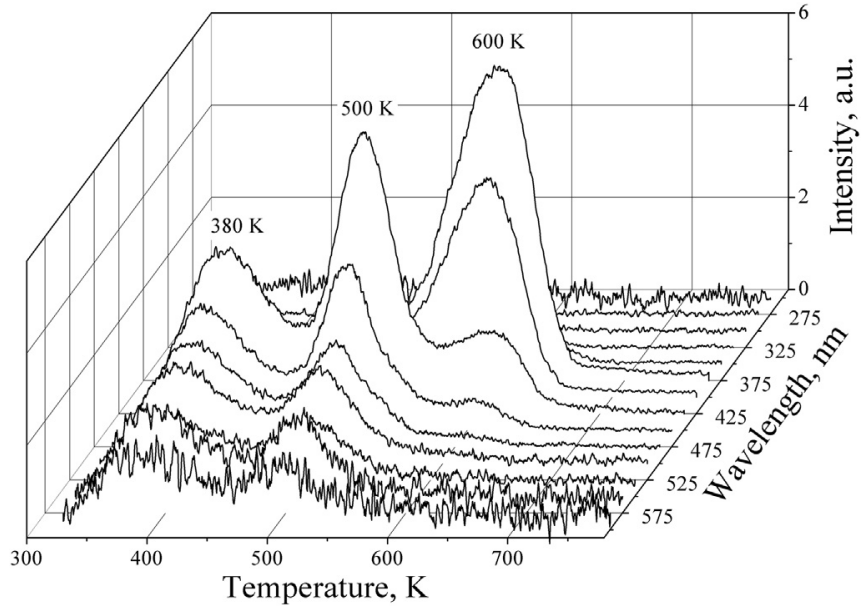

Fig. 2. The TL glow curves measured in different wavelengths for UV irradiated h-BN. peak $600 \mathrm{~K}$ prevails. Within the spectral range under study the intensity of thermally stimulated luminescence is very weak for $\lambda>575$ and $\lambda<300$.

Fig. 3 shows spectral dependences of the observed TL. Here two explicit emission bands can be distinguished, viz.: the $2.90 \mathrm{eV}$ (428 nm) band dominates in the TL spectra of peaks P1 and P2; high-temperature peak P3 is characterized by the $3.25 \mathrm{eV}(382 \mathrm{~nm})$ emission band. Similar bands can be distinguished on temperature sections drawn according to the data of Fig. 2 (see symbols Fig. 3). In addition, analyzing the obtained isothermal sections it can be assumed the presence of weak luminescence in $2.5 \mathrm{eV}$ region.

The TL processes of hot-pressed boron nitride samples were studied by Katzir et al. (1975). It was established that the dominating thermoactivated luminescence is observed within the 360$470 \mathrm{~nm}(2.64-3.44 \mathrm{eV})$ range after ionizing irradiation (UV, X-ray, and $\gamma$ ). Nevertheless, after the complementary EPR, thermally stimulated currents and phosphorescence studies, the authors concluded that the carbon impurity play the role of a recombination center during TL.

Galanov et al. (1989) observed a wide band with the $1.97 \mathrm{eV}$ $(630 \mathrm{~nm})$ maximum in the TL spectrum of the pyrolitic BN after Xray radiation, which was attributed to recombination of quasi-free holes with electrons in deep donor centers. The origin of these centers was not discussed. It should be noted that in the spectral region under study the defects related with technological impurities of the h-BN powders (Watanabe et al., 2004; Weinstein et al., 2011) are optically active. In this case the luminescent complexes with carbon, oxygen as well as structural imperfections with sp3 hybridized bond type may play the dominant role (Zhu et al., 2004).

The spectral bands obtained in this work are in good agreement with independent data on cathodoluminescence $(\mathrm{CL})$ of hexagonal boron nitride nanotubes (Zhi et al., 2005). The observed CL peak in this case was composite and looked like a dominant $3.26 \mathrm{eV}$ maximum with the shoulder in the low-energy region. The specified luminescence was caused by vacancy defects in anion and cation sublattices of h-BN (Zhi et al., 2005).

In Ref. (Tang et al., 2007) the $2.72 \mathrm{eV}$ and $3.33 \mathrm{eV}$ components were observed in the CL spectra of the h-BN nanotubes with various content of oxygen impurity. The authors made a conclusion

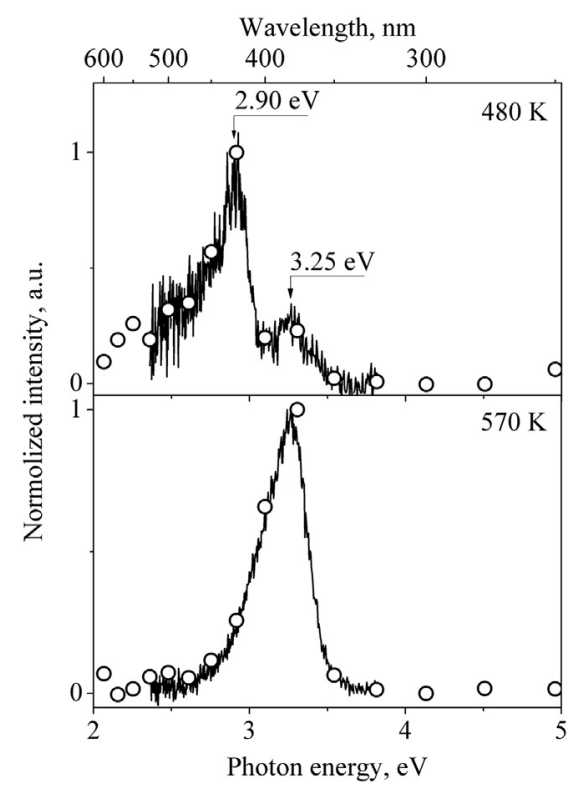

Fig. 3. The TL spectra for the h-BN powder measured at different temperatures. Solid lines - measurements with $800 \mathrm{~nm} / \mathrm{min}$ scanning rate; open circles - isotemperature sections obtained from Fig. 2 
concerning the nature of the observed emission based on the analysis of $\mathrm{CL}$ in the initial pure h-BN, after oxidation due to hightemperature oxygen annealing and subsequent reduction in hydrogen. The $2.72 \mathrm{eV}$ maximum was caused by the centers based on nitrogen and boron vacancies, while the $3.33 \mathrm{eV}$ luminescence was connected with charged $\mathrm{BO}^{-}-$complexes. Thus, we may speak about the dominant role of the oxygen centers and two types of vacancies for luminescent activity of the hexagonal boron nitride within the visible spectral range.

For quantitative analysis of the TL curves we used a formal equation of the general-order kinetics (Chen and McKeever, 1997):

$I(T)=s^{\prime \prime} \cdot n_{0} \cdot \exp \left(-\frac{E_{\mathrm{A}}}{k T}\right) \cdot\left(1+\frac{s^{\prime \prime} \cdot(b-1)}{r} \cdot \int_{T_{0}}^{T} \exp \left(-\frac{E_{\mathrm{A}}}{k \theta}\right) \mathrm{d} \theta\right)^{\frac{b}{1-b}}$

where $n_{0}$ is the trap electron concentration at the initial time, $\mathrm{cm}^{-3}$; $\mathrm{s}^{\prime \prime}$ is the effective frequency factor, $\mathrm{s}^{-1} ; E_{A}$ is the activation energy, $\mathrm{eV} ; k$ is the Boltzmann constant, $\mathrm{eV} / \mathrm{K} T$ is the sample temperature, $\mathrm{K} T_{0}$ is the initial temperature, $\mathrm{K} b$ is the kinetics order, $r$ is the heating rate, $\mathrm{K} / \mathrm{s}$.

All the measured TL curves were described with Eq. (1). The calculated values of model parameters for obtained TL components are given in Table 1 . It is shown that all the TL curves are superpositions of three main components, i.e.: P1, P2 and P3. Fig. 4 depicts approximation of the obtained data for the $375 \mathrm{~nm}$ and $425 \mathrm{~nm}$ emissions. It is seen that the calculated curves describe the experimental results with high accuracy $\left(R^{2}=0.998\right)$. It is evident that the TL curve in the $375 \mathrm{~nm}$ band has very weak peaks at $380 \mathrm{~K}$ and $500 \mathrm{~K}$. It should be noted that a single high-temperature P3 peak can be useful informative signal for applications in the solid state TL dosimetry. To check this assumption it is necessary to study the corresponding dosimetric dependences and to determine low doses sensitivity of h-BN in particular.

According to data analysis of the Table 1, temperature intermediate P2 peak is, nevertheless, related to the deepest trap with $E_{\mathrm{A}}>1.4 \mathrm{eV}$. Bleaching of this trap at lower temperature compared to peak $\mathrm{P} 3\left(E_{\mathrm{A}} \approx 1 \mathrm{eV}\right)$ is due to a higher value of the effective frequency factor (Table 1). In turn, P1 and P3 have similar effective frequency factors, thus pointing to similar nature of the traps responsible for these peaks. It is to be noted that kinetic parameters for peak P1 and P3 well agree with the independent TL data in Refs. (Katzir et al., 1975; Galanov et al., 1989). For example, Ref. Katzir et al. (1975) reports the presence of three TL peaks. It is also shown that two components $380 \mathrm{~K}$ and $700 \mathrm{~K}$ are due to the traps with $E_{\mathrm{A}}=0.7$ and $1.0 \mathrm{eV}$, respectively. Investigation of $\mathrm{TL}$ in pyrolytic h-BN with fractional heating technique stated the presence of three types of traps with activation energies $E_{\mathrm{A}}=0.55,0.85$, and $1.05 \mathrm{eV}$ (Galanov et al., 1989). The values of frequency factors $s^{\prime \prime}=10^{6}-10^{9} \mathrm{~s}^{-1}$ obtained in the same paper also agree with the data of the present work. Note that the low values $\mathrm{s}^{\prime \prime}=10^{4}-10^{5} \mathrm{~s}^{-1}$ are also characteristic of TL processes for example in aluminum nitride with oxygen-vacancy centers (Weinstein et al., 2012).

Table 1

Geometric and kinetic parameters of the TL components.

\begin{tabular}{llll}
\hline Parameters & Peak 1 (P1) & Peak 2 (P2) & Peak 3 (P3) \\
\hline$T_{\max }, \mathrm{K}$ & $380-385$ & $500-507$ & $594-610$ \\
$\omega_{T}, \mathrm{~K}$ & $90-100$ & $49-55$ & $72-80$ \\
$\mu_{\mathrm{g}}$ & $0.64-0.66$ & $0.46-0.49$ & $0.40-0.42$ \\
$E_{\mathrm{A}}, \mathrm{eV}$ & $0.66-0.70$ & $1.43-1.70$ & $0.87-1.10$ \\
$\mathrm{~s}^{\prime \prime}, \mathrm{s}^{-1}$ & $10^{7}-10^{8}$ & $10^{13}-10^{16}$ & $10^{6}-10^{8}$ \\
$b$ & $4.1-4.6$ & $2.0-2.4$ & $1.0-1.2$ \\
\hline
\end{tabular}

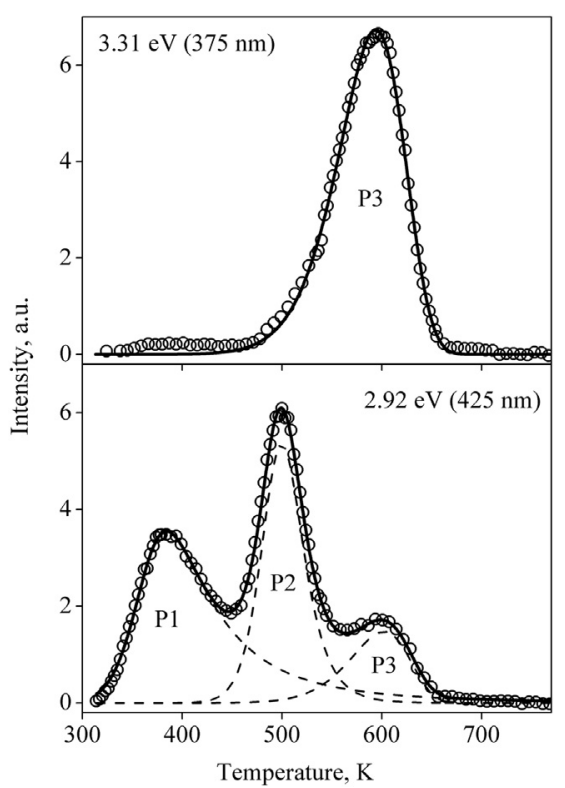

Fig. 4. Analysis of the TL curves with Eq. (1). Symbols - experimental data; dash lines - calculated TL components; solid lines - calculated resulting curves.

Based on the calculated $b$ values (see Table 1 ) one can conclude that the first order processes dominate in the TL kinetics of hightemperature P3 peak and the recombination probability exceeds the charge retrapping. The second order kinetic processes with high retrapping dominate within the P2 temperature range. For P1 peak the academic values $b>4$ are obtained, which have no physical interpretation. This fact may be indicative of the presence of several overlapping TL components in the region of the $380 \mathrm{~K}$ maximum under analysis. Thus, to draw a more reliable conclusion concerning the number of capture centers, emptied within the RT $773 \mathrm{~K}$ temperature range, it is necessary to use additional techniques, e.g. variation of irradiation dose and heating rate, step preheating etc. (Chen and McKeever, 1997). However, these studies are beyond the scope of the present work and at the moment we can mention only three evident components manifested by the maxima on the TL curve.

Bearing in mind the above and guided by the results of Refs. (Katzir et al., 1975), we may conclude that peaks P1 and P3 are caused by luminescence of the related traps based on nitrogen vacancies, e.g., one-boron and three-boron centers. The problem of selecting between electron (Katzir et al., 1975) and hole (Galanov et al., 1989) mechanisms for the TL observed remains open in this case. In turn, the deepest trap responsible for peak P2 is likely to be related with alternative defects. Further spectroscopic studies of hBN are required with control of stoichiometry and impurity content, in particular, oxygen and carbon.

\section{Conclusions}

The features of thermally stimulated luminescence of the hexagonal boron nitride powder exposed to unfiltered UV-radiation are studied. It is shown that two well-defined maxima in the TL spectrum are observed, i.e. $2.90 \mathrm{eV}$ and $3.25 \mathrm{eV}$. On the basis of independent literature data it is suggested that the specified bands are related with luminescence of the vacancy nitrogen-boron $\left(\mathrm{V}_{\mathrm{N}^{-}}\right.$ $\mathrm{B})$ centers and $\mathrm{BO}^{-}$-complexes, respectively.

The TL curves measured in different spectral regions show from one to three components, i.e.: 380,500 and $600 \mathrm{~K}$. All the obtained data were analyzed in terms of the general-order kinetics. Kinetic 
parameters of the 380 and $600 \mathrm{~K} \mathrm{TL}$ peaks point to their relation with the traps based on one-boron and three-boron centers with the depth $\approx 0.7$ and $\approx 1.0 \mathrm{eV}$, respectively. Identification of the trap with $E_{\mathrm{A}}>1.4 \mathrm{eV}$ responsible for the $500 \mathrm{~K}$ peak requires additional studies of h-BN with controlled non-stoichiometry and impurity content.

\section{Acknowledgments}

This work was fulfilled in terms of a State contract under the Federal Program "Scientific and Scientific-Pedagogical Staff of Innovational Russia 2009-2013".

\section{References}

Ci, L., Song, L., Jin, C., Jariwala, D., Wu, D., Li, Y., Srivastava, A., Wang, Z.F., Storr, K., Balicas, L., Liu, F., Ajayan, P.M., 2010. Atomic layers of hybridized boron nitride and graphene domains. Nat. Mater. 9, 430-435.

Chen, R., McKeever, S.W.S., 1997. Theory of Thermoluminescence and Related Phenomena. World Scientific, Singapore.

Galanov, Yu.I., Konusov, F.V., Lopatin, V.V., 1989. Trapping centers and recombination in pyrolytic boron nitride. Russ. Phys. J. 32, 926-929.

Katzir, A., Suss, J.T., Zunger, A., Halperin, A., 1975. Point defects in hexagonal boron nitride. I. EPR, thermoluminescence, and thermally-stimulated-current measurements. Phys. Rev. B 11, 2370-2377.
Kubota, Y., Watanabe, K., Tsuda, O., Taniguchi, T., 2007. Deep ultraviolet lightemitting hexagonal boron nitride synthesized at atmospheric pressure. Science 317, 932-934.

Museur, L., Anglos, D., Petitet, J.-P., Michel, J.-P., Kanaev, A.V., 2007. Photoluminescence of hexagonal boron nitride: effect of surface oxidation under UVlaser irradiation. J. Lumin. 127, 595-600.

Museur, L., Feldbach, E., Kanaev, A., 2008. Defect-related photoluminescence of hexagonal boron nitride. Phys. Rev. B 78, 155204-155211. 155204-155208.

Solozhenko, V.L., Lazarenko, A.G., Petitet, J.-P., Kanaev, A.V., 2001. Bandgap energy of graphite-like hexagonal boron nitride. J. Phys. Chem. Solids 62, 1331-1334.

Tang, C., Bando, Y., Zhi, C., Golberg, D., 2007. Boron-oxygen luminescence centres in boron-nitrogen systems. Chem. Commun. 44, 4599-4601.

Trinkler, L., Berzina, B., Benabdesselam, M., Iacconi, P., Botter-Jensen, L., Atobe, K., 2004. Radiation induced luminescence processes in c-BN. Rad. Meas. 38, 615-618.

Watanabe, K., Taniguchi, T., Kanda, H., 2004. Direct-bandgap properties and evidence for ultraviolet lasing of hexagonal boron nitride single crystal. Nat. Mater. 3, 404-409.

Weinstein, I.A., Vokhmintsev, A.S., Minin, M.G., Chaykin, D.V., Kartashov, V.V., Vlasov, A.V., Karabanalov, M.S., 2011. OSL Activity of Hexagonal Boron Nitride Powders. In: The Spectroscopy and Spectrometry Problems, vol. 28. UrFU, Yekaterinburg, pp. 38-43 (in Russian).

Weinstein, I.A., Vokhmintsev, A.S., Spiridonov, D.M., 2012. Thermoluminescence kinetics of oxygen-related centers in AlN single crystals. Diamond Relat. Mater. 25, 59-62.

Zhi, C., Bando, Y., Tang, C., Golberg, D., Xie, R., Sekigushi, T., 2005. Phonon characteristics and cathodolumininescence of boron nitride nanotubes. Appl. Phys. Lett. 86, 213110-213112

Zhu, Y.-Ch., Bando, Y., Xue, D.-F., Sekiguchi, T., Golberg, D., Xu, F.-F., Liu, Q.-L., 2004. New boron nitride whiskers: showing strong ultraviolet and visible light luminescence. J. Phys. Chem. B 108, 6193-6196. 